Chapter 17

\title{
The Antiagregant Treatment After Coronary Artery Surgery Depending on Cost - Benefit Report
}

\author{
Luminita Iliuta \\ Additional information is available at the end of the chapter \\ http://dx.doi.org/10.5772/54467
}

\section{Introduction}

Despite routine use of ASA before CABG, and lifelong following the revascularization, patients who undergo $C A B G$ remain at high risk of long-term events in any vascular bed (cerebrovascular, cardiovascular, peripheral). The handicap of management of antiplatelet agents in the perioperative period of cardiac surgery requires close collaboration between cardiologists, surgeons and anaesthesiologists. It is necessary to avoid thrombotic complications maintaining the antiagregation, but balancing bleeding complications. [1]

Combined antiplatelet therapy employing agents from different pharmacological classes is characterised by good safety and efficacy profiles.

Antiplatelet therapy and antithrombin therapy have been demonstrated to reduce the risk of cardiac events in patients presenting with acute coronary syndrome, yet all effective therapies also increase the risk of bleeding. Antiplatelet therapy and antithrombotic therapy have been demonstrated to favorably modify clinical outcome, and recent trials of revascularization in ACSs have demonstrated a reduction in the frequency of major cardiac events.[2-14]

Multiple clinical trials showed the favorable benefit/risk ratio of clopidogrel over aspirin justifying the indication for using clopidogrel in a wide range of at risk patients and in longterm prevention in various manifestations of atherosclerosis.[2-9]

Antiplatelet and antithrombin therapy can have synergistic actions that reduce the risk of spontaneous or revascularization, especially percutaneous coronary intervention (PCI)related events. On the other hand, all effective antithrombotic agents also increase the risk of bleeding, especially bleeding that results from vascular access or associated with surgery, including coronary artery bypass grafting (CABG). 
The Clopidogrel in Unstable angina to prevent Recurrent ischemic Events (CURE) trial demonstrated that the combination of clopidogrel and aspirin was superior to aspirin alone for patients hospitalized with non-ST-elevation ACSs.[5] The therapy was in addition to the current standard of care, including heparin or low-molecular-weight heparin, antianginal therapy, and revascularization.[5, 6, 15].

Actually the field of the indications of use of the Clopidogrel is being continuously updated. There are different type of patients who benefit from antiplatelet therapy $[16,17]$ Moreover the combination of two antiagregant drugs (mainly ASA and clopidogrel) in high risk patients is a practice more and more extended [18] and dual antiplatelet therapy is recommended and has to be maintained at least 12 months after drug eluting stent placement [19].

On the other hand, in patients undergoing coronary artery bypass grafting, immediate postoperative antiagregant regimens are only regulated for routinely use Aspirin.

Antiplatelet therapy is critical in the management of coronary artery disease. For patients undergoing coronary artery bypass graft surgery (CABG), controversy remains regarding the safety of preoperative antiplatelet therapy and the optimal postoperative antiplatelet regimen to maintain graft patency and reduce ischemic complications.

Despite $>30$ years of experience with antiplatelet agents during CABG, questions remain regarding their perioperative safety and efficacy. The results of continuing randomized controlled trials should further clarify the role of perioperative aspirin and clopidogrel therapy and help redefine the modern antiplatelet management of coronary artery bypass patients.

Following surgery, extensive evidence supports the use of aspirin, in doses of $100-325 \mathrm{mg}$ daily, to be administered in $48 \mathrm{~h}$ postoperatively and continued indefinitely. Less is known regarding the use of clopidogrel following CABG, although it is now recommended as postoperative antiplatelet therapy in patients with recent acute coronary syndromes.[20]

It is very important to identify the optimal timing and dose ofAaspirin following CABG, and to assess the role of postoperative Clopidogrel therapy.

The recommendations regarding the treatment with Clopidogrel in coronary artery sugery do not take into consideration the cost-benefit ratio which reflect the usefulness from economic point of view, probably because of a the complexity of factors of this equation.

\section{Objectives}

1. To compare the efficacy and safety of Clopidogrel with Aspirin and Aspirin plus Clopidogrel in patients undergoing surgical coronary revascularisation in the immediate postoperative period and 1 year after coronary artery bypass grafting depending on the type of the lesion, on the type of the surgical procedure and on the associated risk factors for gastrointestinal bleeding. 
2. To evaluate the importance and utility of antiplatelet therapy with Clopidogrel early postoperatively in the intensive care unit (ICU) for the prevention of postoperative complications

3. To establish the prognostic implications of the type of the perioperative antiagregant regimen in patients with $C A B G$ and to determine which therapy can reduce hospital stay after cardiac surgery and improve the quality of life of these patients.

4. To determine the indications for using Clopidogrel or Aspirin or Aspirin plus Clopidogrel in coronary artery surgery depending on the cost-benefit ratio and its economic implications.

\section{Methods and material}

Randomized,, open label three years clinical trial with open study period, carried out on 1200 pts undergoing coronary artery bypass grafing divided in three parallel groups: Group A: Clopidogrel po 75 mg/day, Group B: Aspirin po 75 mg/day and Group C: Aspirin 75mg plus Clopidogrel $75 \mathrm{mg}$ once daily.

The main phases of the study protocol were: (Figure 1)

- Enrollment phase - there were enrolled one thousand and two hundred patients undergoing $\mathrm{CABG}$, in the immediate postoperative period

- Active treatment phase - after randomisation all patients received antiagregant therapy:

- Group A with Aspirin 75 mg daily

- Goup B with Clopidogrel 75 mg daily

- Group C with combination of Aspirin 75 mg with Clopidogrel 75 mg.

The treatment began the second day postoperatively and lasted no less than 1 year postoperatively.

- follow -up phase - all patients were evaluated clinically and paraclinically daily for the first ten days and at one, three, six months and one year postoperatively. Patients were followed for a minimum of 1 to a maximum of 3 years, regardless of discontinuation of the study drug. Follow-up assessments took place at 1, 3, 6, and 12 months for all patients and at 1,2 and 3 years for patients randomized early in the study.

\section{Eligibility criteria}

The study included all patients undergoing coronary artery bypass grafting, who underwent surgery in an Emergency Institute for Cardiovascular Diseases between January 1st 2008 and May 1st 2011 who did not have the non - eligibility criterias. 
Patients were over the age of 21, and able to provide informed consent and agreed to comply with all protocol-specified procedures.

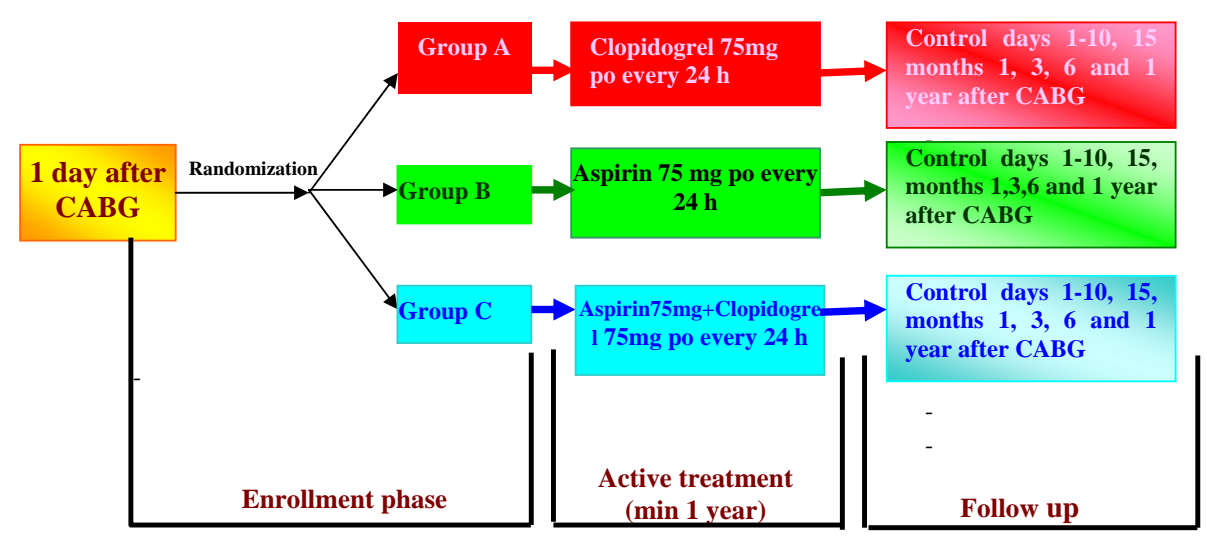

Figure 1. Treatment protocol phases

\section{Non-eligibility criteria}

Patients were excluded from enrolment in the study if any of the following criteria were met:

- Active internal bleeding or risk of hemorrhagic diathesis

- Q-wave myocardial infarction within 24 hours prior to randomization

- Cardiogenic shock.

- Serum Creatinine $\geq 3.0 \mathrm{mg} / \mathrm{dl}$

- severe hepatic failure with ALT or AST > 3x ULN

- Previous use of a GPIIb/IIIa antagonist within 7 days

- Need for long-term anticoagulant or NSAID use

- Failed PCI within 2 weeks prior to randomization

- Active participation in another clinical trial

- Failure to comply with the hospital protocol 


\section{Study drop out criteria}

The occurrence of adverse events (skin reactions, gastrointestinal symptoms, active internal bleeding)

Failure to comply with the hospital protocol/ absence to follow-up

The protocol was approved by the institute management, and every patient signed the informed consent form.

The essential inclusion criteria (gender,mean age, comorbidities, number of grafts per patient, the type of the grafts (arterial or venous) and the mean left ventricular ejection fraction, left ventricular diastolic performance and left atrial dimensions (diameters and area), the duration of treatment and assessment criteria were similar in the three treatment groups $(\mathrm{p}<0.0001)$. All patients received standard therapy including beta blockers, IEC, statins throughout the study period. The patients with exclusive arterial revascularisation also received calcium channel blockers agents but their number was similar in the three groups of study.

Clinical and laboratory parameters were initially assessed, at baseline and at each visit until the end of the study period.

The clinical measurements included: NYHA class for heart failure, presence of angina pectoris, ventricular rhytm, patient compliance and quality of life.

Laboratory parameters included: the usual blood tests (platelet count, hemoglobin, hematocrit, aminotransferases, LDH, biochemistry cholesterol and tryglycerides levels), electrocardiogram(with the evaluation of rhythm, frequence and ST-T elevation), 24 hours ECG Holter monitoring for silent ischemia, stress efort test at 1,3,6 months and 1 year postoperatively and when angina occurred (Bruce or Bruce modified protocol), echocardiography (with assessment of the LV dimensions, ventricular sistolic and diastolic performance, ventricular walls contractility - segmental kinetics, mitral regurgitation degree) and coronarography at 1 year when the other tests where positive for ischemia. Also, at each visit were recorded the occurrence of major and minor bleeding episodes, gastrointestinal symptoms, skin reactions, thrombocytopenia and lab tests abnormalities.

24 hours ECG Holter used a 12 channels monitoring with the evaluation of conduction or rhythm disturbances or occurrence of silent ischemia.

Treadmill stress test was done at 1, 3, 6 months and at 1 year postoperatively and used Bruce or Bruce modified protocol. If the stress test or Holter monitoring diagnosed ischemia at one follow up visit, this was the indication for performing coronarography.

Early development of graft occlusion was diagnosed based on clinical criteria and through electrocardiogram, Holter monitoring, thoracic and transesophageal echocardiography. The appearance of gastrointestinal bleeding was diagnosed using clinical evaluation, endoscopy and colonoscopy. 


\subsection{Primary and secondary endpoints}

The looked at all-cause mortality and major cardiac events, namely cardiac mortality, myocardial infarction or need for target lesion revascularization. The most important endpoints used for the estimation of the medium term prognosis were:

The primary endpoint (efficacy endpoint) was a composite outcome cluster of 30-day mortality, myocardial infarction, in-hospital and at 1 year occurrence of graft occlusion (efficacy endpoints), total hospital stay and immobilization (measured in days), Intensive Care Unit length of stay and cost, quality of life. Quality of life was appreciated using a scale from one to ten calculated on the base of a questionnaire filled by the patients at each visit

The secondary endpoints at 30 days looked at in-hospital major peripheral or bleeding complications (including surgical bleeding complications, transfusion of at least two units of blood, intracranial bleeding, retroperitoneal bleeding, overt hemorrhage), neutropenia $(<1.5 \mathrm{x}$ 109 per litre), thrombocytopenia ( $<100 \times 109$ per litre), early discontinuation of the study drug due to a non-cardiac adverse event (including death of non-cardiac origin) (safety endpoint).

The data collected represented the fields of a database in the Visual Fox Pro computer program. Data were processed by means of computers, using the Excel, EpiInfo, Systat and SPSS programs for multivariate regression analysis and relative risk and correlation coefficient calculation

No confirmatory statistical hypothesis was pre-specified, but a detailed analysis plan was defined before the database was locked. This analysis plan was based on generating risk ratios and $\mathrm{CIs}$ ( $\mathrm{CI}=$ confidence index) for the pairwise comparisons of primary interest. These comparisons were presented with the two - sided $95 \%$ CI of the relative risk and with normal $\mathrm{p}$ values. For the primary endpoints Kaplan-Meier curves were constructed and log-rank tests were done. For each endpoint, a two-sided 95\% CI was also calculated and an overall Chi square test, comparing the three treatment groups was done [19, 21, 25].

The frequency of the primary efficacy plus safety endpoint for the Aspirin group as a reference group was $17,7 \%$. On the basis of phase-II studies we assumed that the experimental groups with Clopidogrel and Aspirin plus Clopidogrel would result in better, or at least similar outcomes when compared with standard treatment. The sample size and power calculations were therefore based on non-inferiority of the experimental group versus the reference group. The study has $80 \%$ power to exclude, with $95 \%$ confidence (one-sided), a $1 \%$ higher rate of the primary endpoints compared with the reference group, provided the point estimate in the experimental treatment group was 1,7\% lower for the efficacy endpoint and $2 \%$ lower for the efficacy and safety endpoint. [2-11, 13-18, 22]

\section{Patients}

The study included 1200 patients undergoing coronary artery bypass grafing with arteries (internal mammar, radial, gastroepiploic) or inverted saphenal veins. The patients were 
randomised to receive Clopidogrel $75 \mathrm{mg}$ daily or Aspirin $75 \mathrm{mg}$ daily or Aspirin plus Clopidogrel $75 \mathrm{mg}$ daily one day after surgery and in the postoperative period for no less than 1 year.. The patients undergoing also venticular remodelling for aneurysms were not taken in our study.

The baseline characteristics were similar in the three arms of the study (Table 1). Overall, the study populations were similar to those of previous trials on antiagregants.

\begin{tabular}{|c|c|c|c|}
\hline & $\begin{array}{c}\text { Group A - } \\
397 \text { pts }\end{array}$ & $\begin{array}{c}\text { Group B- } 401 \\
\text { pts }\end{array}$ & $\begin{array}{l}\text { Group C- } \\
402 \text { pts }\end{array}$ \\
\hline Mean (SD) age (years) & $62,3(12)$ & $62,5(13)$ & $62,4(12)$ \\
\hline Age"/>70 years & $13,85 \%$ & $14,21 \%$ & $14,43 \%$ \\
\hline Women & $25,94 \%$ & $26,18 \%$ & $26,62 \%$ \\
\hline Family history of heart disease (\%) & $49,62 \%$ & $50,12 \%$ & $49,75 \%$ \\
\hline Dislipidemia (\%) & $75,06 \%$ & $75,81 \%$ & $76,37 \%$ \\
\hline Prior myocardial infarction (\%) & $33,50 \%$ & $33,91 \%$ & $34,58 \%$ \\
\hline NYHA class "/>II & $20,15 \%$ & $20,70 \%$ & $20,89 \%$ \\
\hline Prior stroke (\%) & $6,29 \%$ & $6,73 \%$ & $6,96 \%$ \\
\hline Peripheral arterial disease & $9,82 \%$ & $9,72 \%$ & $10,45 \%$ \\
\hline Atrial fibrillation & $6,04 \%$ & $6,48 \%$ & $6,47 \%$ \\
\hline Hypertension & $65,49 \%$ & $66,58 \%$ & $64,92 \%$ \\
\hline Diabetes mellitus & $25,19 \%$ & $25,43 \%$ & $25,12 \%$ \\
\hline Current smoker & $26,45 \%$ & $26,43 \%$ & $25,87 \%$ \\
\hline Re-intervention (previous coronary artery surgery) & $8,82 \%$ & $8,98 \%$ & $8,95 \%$ \\
\hline
\end{tabular}

Table 1. Baseline characteristics

The medications used chronically by the patients at the time of randomization were similar in the Aspirin, Clopidogrel and Aspirin plus Clopidogrel treatment arms and are are listed in Table 2 


\begin{tabular}{llll}
\hline & $\begin{array}{c}\text { Group A - } \\
\text { 397 pts }\end{array}$ & Group B- 401 pts & Group C- \\
& $23,68 \%$ pts \\
\hline Digoxin & $67,25 \%$ & $23,94 \%$ & $24,13 \%$ \\
\hline ACE inhibitors & $24,43 \%$ & $68,58 \%$ & $63,68 \%$ \\
\hline Angiotensin Il inhibitors & $89,92 \%$ & $23,69 \%$ & $25,12 \%$ \\
\hline Beta blockers & $61,46 \%$ & $89,28 \%$ & $90,29 \%$ \\
\hline Aspirin before surgery & $25,44 \%$ & $63,84 \%$ & $65,17 \%$ \\
\hline Calcium channel blockers & $19,90 \%$ & $25,93 \%$ & $26,37 \%$ \\
\hline Diuretics & $21,91 \%$ & $20,70 \%$ & $20,39 \%$ \\
\hline Aldactone & $89,92 \%$ & $20,89 \%$ \\
\hline Lipid lowering agents & & & \\
\hline
\end{tabular}

Table 2. Number of patients who received concomitant medications during stay in hospital

$61,46 \%$ of patients received Aspirin before surgery in group A, respectively 63,84\% in group $\mathrm{B}$ and $65,17 \%$ in group $\mathrm{C}$.

The primary efficacy and efficacy plus safety endpoints and their individual components in the treatment groups are shown in Table 3.

The clinical diagnosis at the time of randomization was similar in the three treated arms of the study:

- Over half of the patients presented with unstable angina (49,62\% in group A, $51,63 \%$ in group $\mathrm{B}$ and $53.48 \%$ respectively in group C).

- Approximately one in five-six patients had experienced a recent myocardial infarction (16.37\% in group A, $21,94 \%$ in group B and $22.39 \%$ respectively in group C).

- About a third presented with stable angina or another diagnosis requiring antiagregant regimen (aproximatively 33,6\% in each treatment arm - 33.75\% in group A, 33,66\% in group B, 33,58\% in group C).

\section{Statistics (Figure 2, 3)}

The data base was done using Visual Fox Pro programme. The main variables used were: 
- Prediction variables :

- patient ID Data

$\circ$ preoperative diagnosis

- surgical risk (calculated using a scale from 1 to 10 taking into account different preoperative parameters: age, co-morbidities, severity of cardiac lesions (NYHA class), type and duration of surgical intervention, associated risk factors)

- type of surgical intervention

- specific variables related to the surgical performance: duration of surgical intervention, intraoperative complications

- ICU duration and complications occured

- Outcomes variables:

- presence and type of postoperative complications

$\circ$ death and its causes.

The statistical analysis was performed using the SYSTAT and SPSS programmes for:

- Measurement of the power of association between the prediction variables and outcomes using different tests depending on the type of variables:

- for qualitative variables: CHI square test or Fischer exact test

- for quantitative variables: $\mathrm{T}$ test (Student test), ANOVA test or $\mathrm{U}$ test depending on samples volumes and Kruskal Wallis nonparametric tests or other methods of statistical correlation as analysis of simple linear and multivariate regression

- Relative Risk calculation and the 95\% confidence limits for treatment groups

- Cost-benefit ratio calculation for using different antiplatelets agents after coronary artery bypass grafting. It was determined using a special programme, which used the data from the database and different economic data from specialized departments from our Institute, in order to perform the assessment of the efficiency of different antiplatelet therapies following coronary artery surgery.

The calculation of the cost-benefit ratio for each type of treatment and for routinely use clopidogrel in CABG was done taking into account the following parameters:

- parameters related to the type of the treatment

$\circ$ cost of the treatment for each patient

- number of supplementary echographic and endoscopic examinations per patient

- number of bleeding episodes and cost per patient

- global cost/ patient 
- parameters related to surgical intervention

- early postoperative mortality rates for surgical intervention (global and specific depending on individual risk and type of the antiagregant regimen)

$\circ$ in hospital and at 1 year graft occlusion/myocardial infarction/severe bleeding on subgroups of patients taking into account the individual risk

- immediate and long term postoperative complications rates depending on the type of the antiagregant regimen

- ICU length of stay and cost

- quality of life at 1 month and 1 year postoperatively on risk subgroups and on type of surgical interventions depending on the type of the antiagregant regimen

- Parameters related to the patient

$\circ$ age

$\circ$ gender

- co-morbidities

○ associated risk factors.

Using the above mentioned parameters, the special programme calculated a risk score per patient on types of treatment and the cost of routinely use clopidogrel in cabg patients, which was used then for estimation of the cost-benefit ratio associated with the type of the antiagregant regimen

Data were grouped on types of surgical interventions according to the exposure level to the risk factors. For each exposure level there were introduced the number of patients taking Clopidogrel (cases) and the number of patients who have not taken Clopidogrel (controls). The confounders were controlled by stratification.

Data interpretation was performed taking into account the following hypothesis:

- a cost-benefit report $>1$ was considered unfavourable from economic point of view; for these patients the routine use of Clopidogrel as antiplatelet therapy after coronary artery bypass surgery was considered as having uncertain indication;

- a cost-benefit report $=1$ was considered neutral and included the patients subgroups classified as relative indication for the routine use of clopidogrel as antiplatelet therapy after coronary artery bypass surgery, risks and benefits of using that therapy it being appreciated on case to case basis, depending on the risk and benefit for each patient;

- a cost-benefit report $<1$ was considered favourable from economic point of view; for these patients the routine use of Clopidogrel as antiplatelet therapy after coronary artery bypass surgery was considered as having a standard indication, being recommended in each case. 


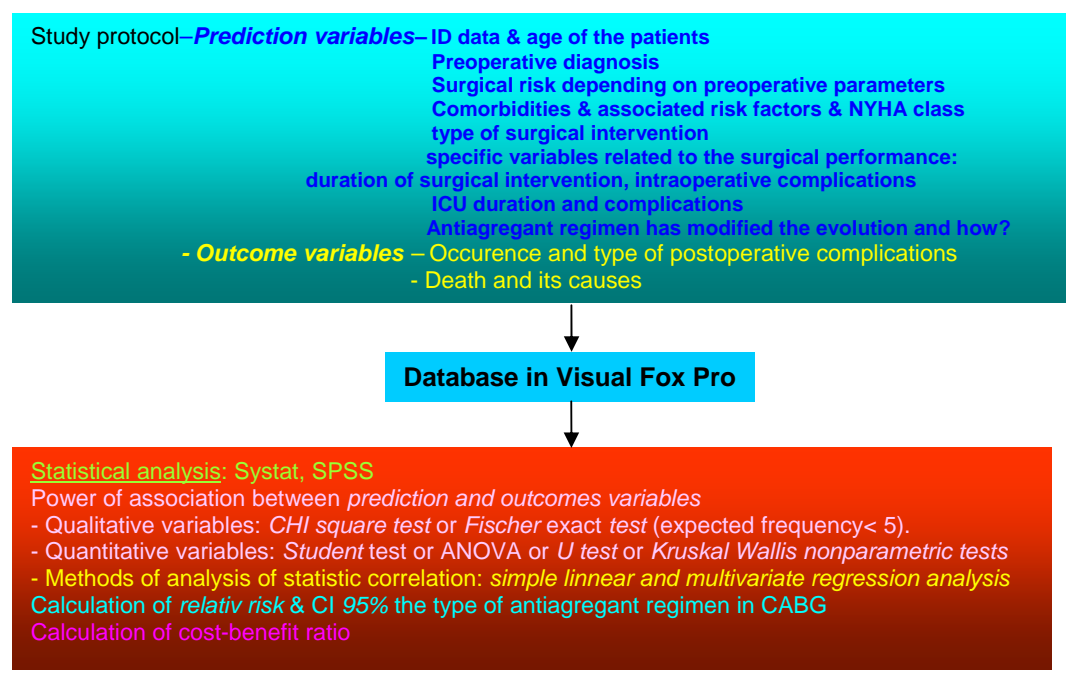

Figure 2. Statistic methodology

Cost-benefit ratio calculation for routinely use Clopidogrel postCABG $\Rightarrow$ Parameters related to:

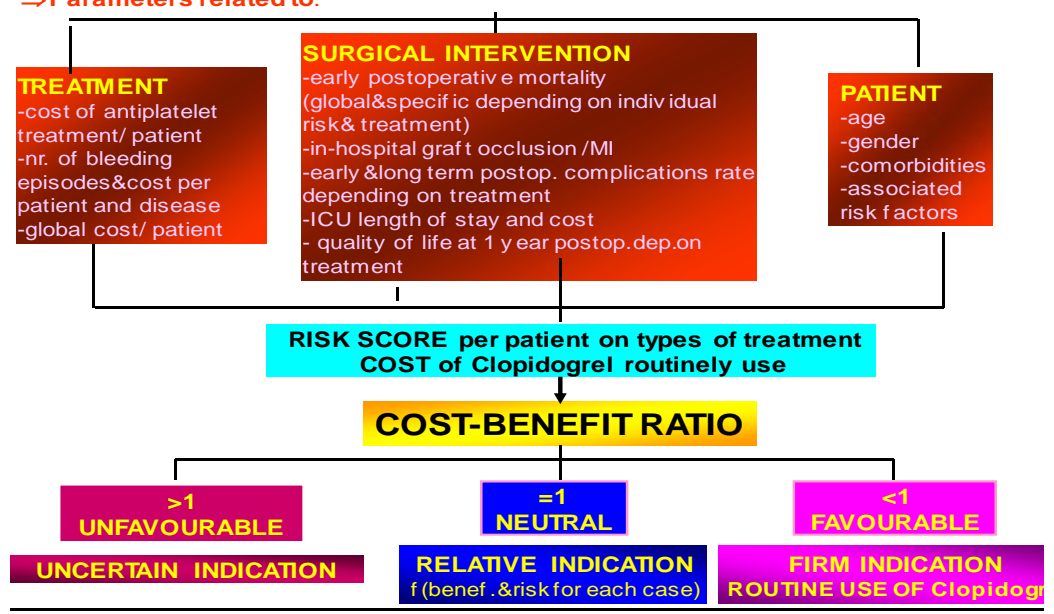

Figure 3. Statistical analysis and cost-benefit report calculation 


\section{Results}

The main conclusion of our study was that using Clopidogrel single or associated with Aspirin for antiplatelet treatment in the immediate postoperative period in CABG patients is more effective than Aspirin alone, with a better cost-benefit report. The cost benefit report associated with using Aspirin plus Clopidogrel was almost two times higher than with Aspirin alone (Figure 4)

The incidence of myocardial infarction and death following graft thrombosis was $21 \%$ in Aspirin group, $12 \%$ in Clopidogrel group and respectively $7 \%$ in aspirin plus Clopidogel group.
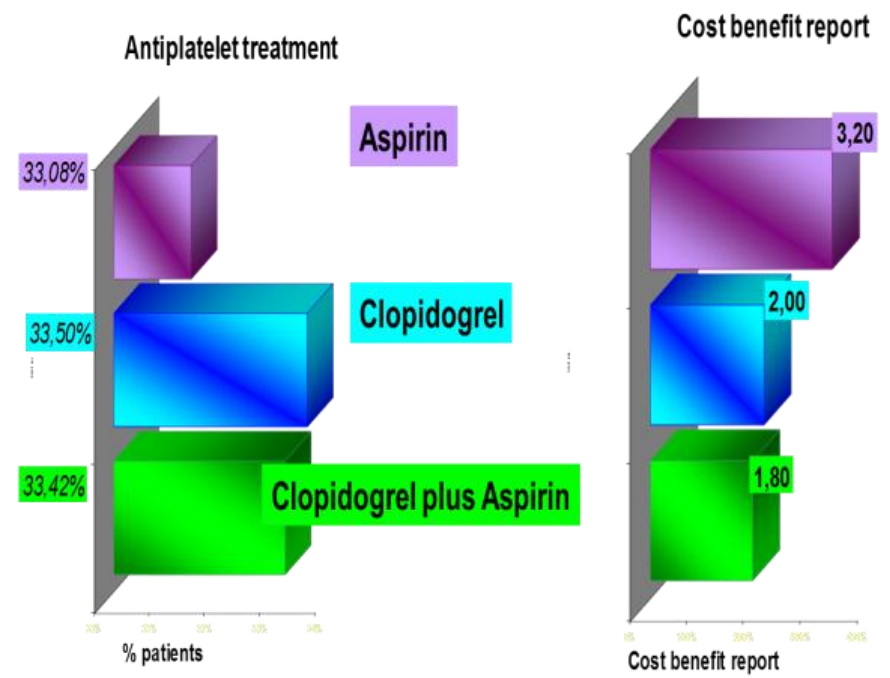

Figure 4. Cost-benefit report depending on the type of antiplatelet treatment in CABG patients

Relative risks and 95\% confidence indexes for primary efficacy composite endpoints (30 days mortality, myocardial infarction, inhospital graft oclusion, hospital stay and immobilization (days), Intensive Care Unit length of stay and cost, quality of life) were different depending on the patients age, NYHA class, LVEF, the severity of associated MR, but, in all cases were lower among patients treated with Clopidogrel associated with Aspirin than among those treated with Aspirin alone

Also, there were different depending on the patients age, NYHA class, LVEF and associated severe mitral regurgitation.

Conventional statistical testing for Clopidogrel plus Aspirin versus Clopidogrel alone versus Aspirin alone resulted in $p$ values of 0,0002 and 0,0003 respectively for the primary efficacy plus safety composite endpoints. 


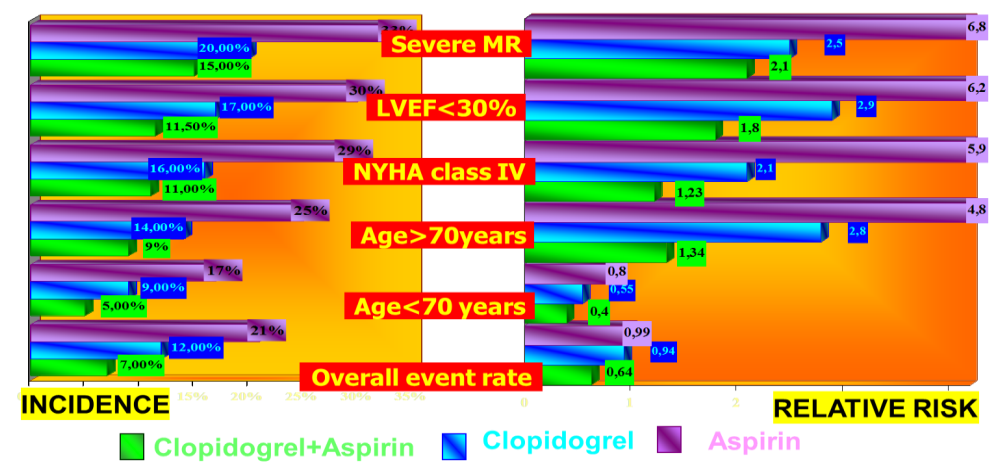

Figure 5. Relative risks and 95\% Confidence Indexes for primary efficacy composite endpoints in the study groups

At hospital discharge and at 30 days, the combined efficacy and safety outcome endpoints were smaller in Clopidogrel plus Aspirin group.

For the primary efficacy plus safety endpoint (30 day mortality, inhospital graft oclusion or inhospital major bleeding), the rates were smaller for Clopidogrel plus Aspirin group, as the rates of in-hospital death

In-hospital graft oclusion and myocardial infarction occurred rarely in patients treated with Clopidogrel plus Aspirin compared with the patients treated with Aspirin alone. Major hemorrhagic events were similar in the study groups. Concerning the duration of the hospitalisation and imobilisation, there were a little bit smaller in Clopidogrel plus Aspirin group. (Figure 6)

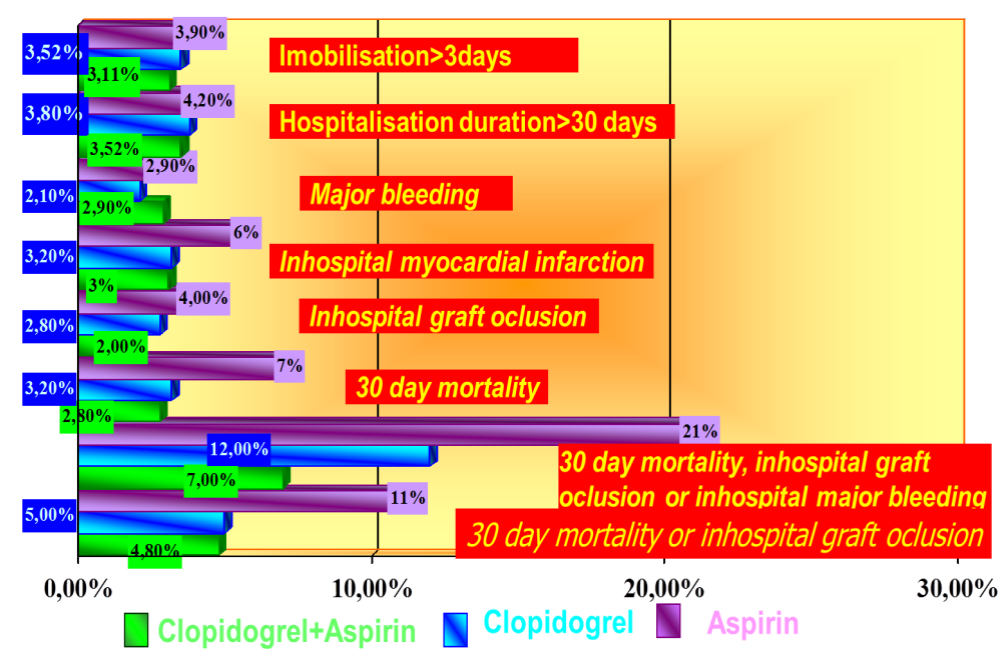

Figure 6. Frequency of composite and single endpoints at hospital discharge and at 30 days 
On long term, the incidence of death, myocardial infarction, and revascularization occurring at one year following CABG was greater in Aspirin group compared with Clopidogrel and Clopidogrel plus Aspirin groups (15\% versus 12\% versus 10\%)

The Kaplan Meier curves for primary efficacy and safety endpoints showed a smaller probability for death, myocardial infarction or graft oclusion in Clopidogrel plus Aspirin group (Figure 7).

Early after treatment, the curves for Clopidogel associated or not with Aspirin started to separate from the one of Aspirin alone. At 30 days, differences in the primary endpoints between the three groups were already present.

Until the end of the follow up, for the primary efficacy endpoint and for the primary efficacy plus safety end point, event rates were abut two times higher for Aspirin group compared with Clopidogrel plus Aspirin group with log rank tests highly significant and significant $\mathrm{p}$ values $(\mathrm{p}<0,0001)$.

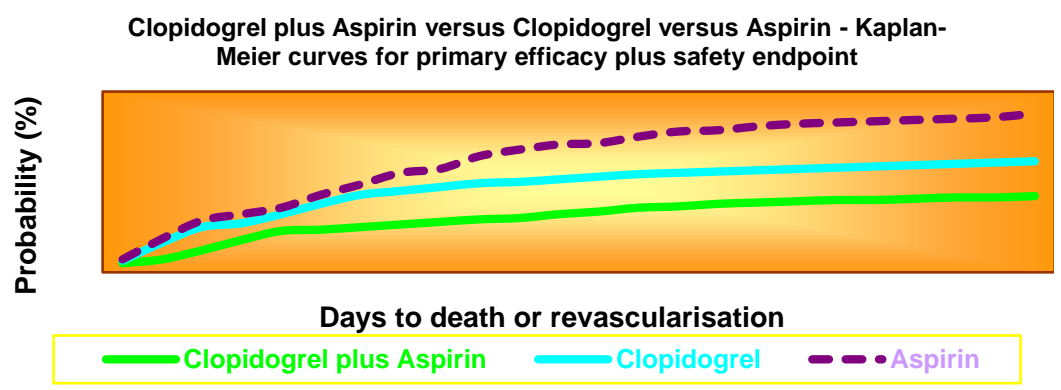

Figure 7. The Kaplan Meier curves for primary efficacy and safety endpoints

Concerning antiagregany therapy complications, the dates on in-hospital strokes are summarized in Figure 8.

There were no significant differences between the three groups regarding major hemorrhage and thrombocytopenia. Minor hemorrhage occurs more frequently in patients taking Aspirin. Total stroke and ischemic stroke rates were similar in the three groups. A few hemorrhagic conversions were seen in each of the tthree treatment groups. More minor or major bleeding complications and blood transfusions were also seen in the aspirin alone or associated with clopidogrel groups compared with clopidogrel alone group, although these differences were not significant.

Significantly more major bleeding complications $(p=0,0001)$, more transfusions $(p=0,002)$ and a higher rate of thrombocytopenia $(\mathrm{p}=0,001)$ were seen in patients with associated treatment with anticoagulants, in patients older than 75 years and in diabetics, the rate of major bleeding complications was three times higher in those with associated anticoagulant therapy (4\%versus $14 \%$ and $2 \%$ versus $7 \%$ respectively 


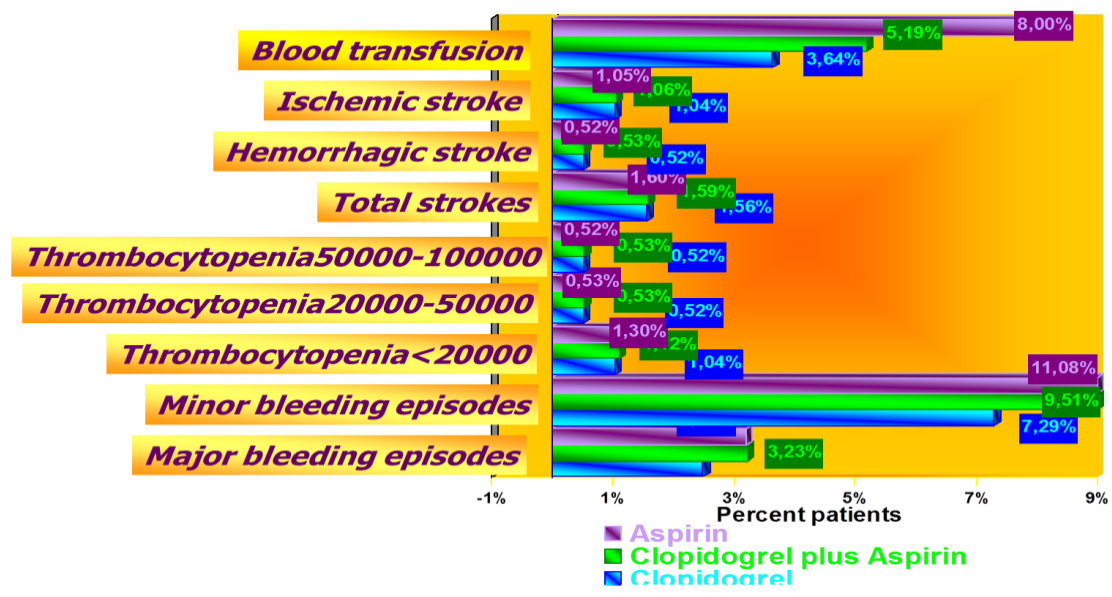

Figure 8. Hemorhagic and ischemic postoperative complications in the study groups.

The probability of early graft oclusion and perioperative myocardial infarction was smaller with Clopidogrel alone or associated with Aspirin versus Aspirin alone, the associated relative risks being negative because the studied drugs worked as protection factors for these perioperative complications. (Figure 9)

As we seen before, the relative risks for the most severe antiagregant therapy complications, hemoragic stroke were similar in the three study groups

Clopidogrel plus Aspirin versus Clopidogrel versus Aspirin relative risk for early graft thrombosis, acute myocardial infarction or hemorrhagic stroke

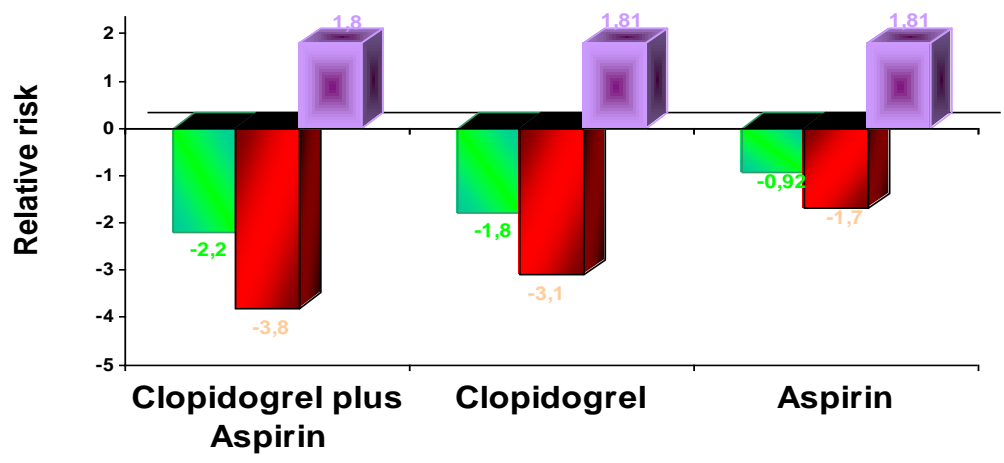

Early graft oclusion $\square$ Acute myocardial infarction $\mathbf{x}$ Hemorrhagic stroke

Figure 9. Relative risk for early graft thrombosis, acute myocardial infarction or hemorrhagic stroke 


\section{Discussions}

Multiple clinical trials showed the favorable effects of Clopidogrel alone or combined with Aspirin extending the indication for using Clopidogrel in a wide range of at risk patients and in long-term prevention in various manifestations of atherosclerosis.

In recent years, enormous growth in the use of coronary stenting procedures has resulted in a significant decrease in restenosis rates, while acute and sub-acute stent thrombosis remain a significant potential complication. It has been shown, however, that the risk of acute and subacute stent thrombosis is greatly reduced by the administration of antiplatelet therapies following stenting. Much clinical experience with combination of aspirin and ticlopidine has been gained, however ticlopidine has been shown to be associated with rare risk of haematological adverse events.

The CLASSICS study demonstrated the safety and efficacy of clopidogrel (with or without loading dose) in combination with aspirin for use following coronary stenting.

A large randomized trial has demonstrated that the acute administration of clopidogrel-a long-acting antiplatelet therapy - to patients with non-ST-segment elevation acute coronary syndromes (NSTE ACS) can reduce subsequent risk for death, myocardial infarction, or stroke by $20 \%$ when continued for a mean duration of nine months [21]. However, single-center case series have demonstrated that, in patients requiring coronary artery bypass graft surgery, the use of Clopidogrel is associated with increased risk of perioperative bleeding and a need for transfusion [22- 26].

This risk appears to be time dependent. For example, post-hoc data analysis from the CURE (Clopidogrel in Unstable Angina to Prevent Recurrent Events) trial revealed that bleeding risks were increased when patients had CABG surgery within 5 days of clopidogrel treatment but not when surgery was delayed for $>5$ days after treatment with clopidogrel [21]

These findings are reflected in the American College of Cardiology/American Heart Association (ACC/AHA) guidelines for the acute management of patients with NSTE ACS, which endorse the acute use of clopidogrel but also recommend withholding clopidogrel for at least 5 days before CABG surgery (27).

Adherence in community practice to this guidelines recommendation is very unclear. has not been characterized previously. There are studies trying to characterize patterns of Clopidogrel use before CABG and to examine the time-dependent risks for postoperative transfusion among NSTE ACS patients treated at 264 hospitals participating in the CRUSADE (Can Rapid Risk Stratification of Unstable Angina Patients Suppress Adverse Outcomes With Early Implementation of the ACC/AHA Guidelines) National Quality Improvement Initiative [15, 28- 29].

Combined antiplatelet therapy was also studied in a lot of trials and most of them showed good safety and efficacy profiles. Antiplatelet therapy and antithrombin therapy have been demonstrated to reduce the risk of cardiac events in patients presenting with acute coronary syndrome, yet all effective therapies also increase the risk of bleeding. Antiplatelet therapy 
and antithrombotic therapy have been demonstrated to favorably modify clinical outcome, and recent trials of revascularization in ACSs have demonstrated a reduction in the frequency of major cardiac events[2-14].

The benefits versus risks of early and long-term clopidogrel therapy (freedom from CV death, MI, stroke, or life-threatening bleeding) are similar in those undergoing revascularization (CABG or PCI) and in the study population as a whole. Overall, the benefits of starting clopidogrel on admission appear to outweigh the risks, even among those who proceed to CABG during the initial hospitalization.

Actually the field of the indications of use of the antiagregant therapy is being continuously updated.The role of the aspirin in the primary prevention has extended its prescription based on related factors of cardiovascular and/or neurological risk. Moreover the combination of two antiagregant drugs (mainly Aspirin and clopidogrel) in high risk patients is a practice more and more extended [18]. Dual antiplatelet therapy has to be maintained at least 12 months after drug eluting stent placement and, in this patient a specific protocol of antiaggregation in type, combination and duration need to be applied [30,31].

For patients undergoing coronary artery bypass graft surgery, controversy remains regarding the safety of preoperative antiplatelet therapy and the optimal postoperative antiplatelet regimen to maintain graft patency and reduce ischemic complications. There are also of this systematic reviews trying to evaluate the risks and benefits of preoperative aspirin and clopidogrel therapy, to identify the optimal timing and dose of aspirin following CABG, and to assess the role of postoperative clopidogrel therapy.[20]Following surgery, extensive evidence supports the use of aspirin, in doses of 100 - $325 \mathrm{mg}$ daily, to be administered in 48 $\mathrm{h}$ postoperatively and continued indefinitely. Less is known regarding the use of clopidogrel following $C A B G$, although it is now recommended as postoperative antiplatelet therapy in patients with recent acute coronary syndromes.Despite $>30$ years of experience with antiplatelet agents during $C A B G$, questions remain regarding their perioperative safety and efficacy. The results of continuing randomized controlled trials should further clarify the role of perioperative aspirin and clopidogrel therapy and help redefine the modern antiplatelet management of coronary artery bypass patients.

Also, the optimal aspirin dose for the prevention of cardiovascular events remains controversial.[32]: Daily aspirin doses of $100 \mathrm{mg}$ or greater were associated with no clear benefit in patients taking aspirin only and possibly with harm in patients taking clopidogrel. Daily doses of 75 to $81 \mathrm{mg}$ may optimize efficacy and safety for patients requiring aspirin for long-term prevention, especially for those receiving dual antiplatelet therapy.

The response to aspirin and/or clopidogrel and its impact on graft patency after off-pump coronary artery bypass grafting is characterised by individual variability, but, overall combined clopidogrel and aspirin overcome single drug resistances, were are safe for bleeding and improve venous graft patency. [33]

At first sight, clopidogrel appears to be undesirable for cardiac surgeons: antiplatelet therapy can increase the risk of bleeding during coronary artery bypass graft surgery (CABG).1 Traditionally, many surgeons have felt that, with impeccable technique, their personally 
constructed grafts would be nearly 'immune' to thrombosis, even without antiplatelet therapy. However, it could theoretically reduce the risk for early vein graft failure, which is predominantly thrombosis related.

There are three different principal mechanisms that play a role in vein graft failure during postoperative periods: early ( $<1$ month): thrombosis; related to technical factors, Intermediate ( 1 to 12 months): intimal hyperplasia and Later postoperative (>12 months): accelerated atherosclerosis [34]

Concern about possible hemorrhagic complications arising from use of oral antiplatelet agents in immediate proximity to coronary artery bypass graft (CABG) surgery leads many clinicians to avoid or discontinue these agents preoperatively. Recent evidence suggests that the modest hemorrhagic risk may be acceptable, given the clinical benefits of sustained antiplatelet therapy in preventing graft occlusion and ischemic complications pre- and post-CABG. [35]

Also, other analysis provide insight into patterns of clopidogrel use and outcomes in the setting of CABG performed on patients with NSTE ACS [36] and found that as many as $30 \%$ of patients currently receive clopidogrel before CABG surgery, and, of these, nearly $90 \%$ have surgery within 5 days of treatment, contrary to the ACC/AHA guidelines recommendations. These data demonstrating a modest increase in transfusion risk in part reflect a more stable estimate of risks based on a much larger case sample in the CRUSADE Initiative.

The benefits versus risks of early and long-term clopidogrel therapy (freedom from CV death, MI, stroke, or life-threatening bleeding) were similar in those undergoing revascularization (CABG or PCI) and in the study population as a whole. Overall, the benefits of starting clopidogrel on admission appear to outweigh the risks, even among those who proceed to CABG during the initial hospitalization.[26]

Data from the Antiplatelet Trialists' Collaboration support the use of antiplatelet therapy (mostly data for aspirin) after CABG and further data support the initiation of aspirin within 48 hours of CABG. The CURE trial provides the opportunity to explore the combined use of aspirin and clopidogrel for those undergoing CABG.[26]

Clopidogrel offers multiple advantages in acute and chronic use in coronary intervention. The favorable benefit/risk ratio of clopidogrel over aspirin established by CAPRIE, combined with its characteristics related to rapid onset of action, loading dose, pre-treatment efficacy and ease of use, justify the consideration of using clopidogrel in a wide range of at risk patients and in long-term prevention in various manifestations of atherosclerosis / atherothrombosis.

Combined antiplatelet therapy employing agents from different pharmacological classes after CABG was characterised by good safety and efficacy profiles. The absence of interaction, and the potential synergistic effect when used with other antithrombotic agents, will allow clinicians to optimise treatment in acute situations. Combination therapy, using clopidogrel and other drugs commonly administered for a range of cardiovascular and other disorders, appears safe after CABG.

Despite routine use of ASA before CABG, and lifelong following the revascularization, patients who undergo $\mathrm{CABG}$ remain at high risk of long-term events in any vascular 
bed (cerebrovascular, cardiovascular, peripheral). The incidence of death, MI, and revascularization occurring at one and three-year following a CABG is greater than $15 \%$. 3. Therefore, patients who undergo CABG could benefit from long-term therapy that provides improved protection against all types of atherothrombotic events such as myocardial infarction, ischemic strokes, and vascular death.

\section{Study limitations}

First, our comparisons of clinical outcomes by treatment strategy were observational. Although we adjusted all comparisons for baseline clinical factors, we cannot exclude any persistent unmeasured confounding. Nonetheless, because a randomized clinical trial evaluating the benefits and risks of different antiagregant regimen of patients undergoing CABG is unlikely to be undertaken, this study is the first to provide insight into the scope of this issue at a national level.we considered the diagnostic of ischemia using stress test, Holter monitoring and, in case of a positive result, invasive coronarography as sufficient. Second, we did not collect data on the incidence of re-exploration at 2 or three years after CABG, although we had some information about that and we did nor perform routinely coronarography at 1 year postoperatively to all patients.

\section{Conclusions}

1. Antiplatelet therapy with Clopidogrel plus Aspirin in the immediate postoperative period in patients with $\mathrm{CABG}$ was associated with an better cost-benefit report, proving to be more effective than Aspirin alone.

Taking into account both efficacy and safety, the combined antiplatelet therapy with Clopidogrel and Aspirin emerged as the best treatment in this trial.

2. The favourable cost/benefit ratio of Clopidogrel over Aspirin established by this study, combined with its characteristics related to rapid onset of action, loading dose, pretreatment efficacy and ease of use, justify the consideration of routinely using Clopidogrel in CABg patients and in long-term prevention in various manifestations of atherosclerosis

3. Taking into account cost-benefit report when comparing antiplatelet strategies after CABG,treatment with Aspirin alone was associated with an cost benefit report almost 1 in terms of reducing mortality and graft oclusion, Clopidogrel alone with a little bit more than one and the asociated therapy had an cost benefit ratio about 3, emerged as the best treatment inthis trial. It should be regarded as an attractive alternative pharmacological antiplatelet strategy in the immediate postoperative period in CABG patients,deserving further studies 


\section{Acknowledgements}

Special thanks to cardiac surgeons and anesthesiologists from the Emergency Institute for Cardiovascular Diseases "C.C.Iliescu”, Bucharest, Romania

\section{Author details}

Luminita Iliuta

University of Medicine and Pharmacy “Carol Davila" - Bucharest, Romania

\section{References}

[1] Ferrandis, R, Llau, J. V, \& Mugarra, A. Perioperative Management of AntiplateletDrugs in Cardiac Surgery; Curr Cardiol Rev. (2009). May; , 5(2), 125-132.

[2] Collaborative meta-analysis of randomized trials of anti-platelet therapy for prevention of deathmyocardial infarction, and stroke in high risk patients: Anti-Platelet Trialists' Collaboration. BMJ. (2002). Abstract/FREE Full Text, 324, 71-86.

[3] Kong, D. F, Califf, R. M, Miller, D. P, et al. Clinical outcomes of therapeutic agents that block the platelet glycoprotein IIb/IIIa integrin in ischemic heart disease. Circulation. (1998). Abstract/FREE Full Text, 98, 2829-2835.

[4] Boersma, E, Harrington, R. A, Moliterno, D. J, et al. Platelet glycoprotein IIb/IIIa inhibitors in acute coronary syndromes: a meta-analysis of all major randomised clinical trials. Lancet. (2002). CrossRefMedline, 359, 189-198.

[5] Clopidogrel in Unstable Angina to Prevent Recurrent Events Trial InvestigatorsEffects of clopidogrel in addition to aspirin in patients with acute coronary syndromes without ST-segment elevation. N Engl J Med. (2001). CrossRefMedline, 345, 494-502.

[6] Mehta, S, Yusuf, S, et al. Effects of pre-treatment with clopidogrel and aspirin followed by long-term therapy in patients undergoing percutaneous coronary intervention: the PCI-CURE study. Lancet. (2001). CrossRefMedline, 358, 527-533.

[7] PURSUIT Trial InvestigatorsInhibition of platelet glycoprotein IIb/IIIa with eptifibatide in patients with acute coronary syndromes. N Engl J Med. (1998). CrossRefMedline, $339,436-443$.

[8] PRISM Study InvestigatorsA comparison of aspirin plus tirofiban with aspirin plus heparin for unstable angina. N Engl J Med. (1998). CrossRefMedline, 338, 1498-1505.

[9] GUSTO-IV ACS InvestigatorsEffect of glycoprotein IIb/IIIa receptor blocker abciximab on outcome in patients with acute coronary syndromes without early coronary 
revascularization: the GUSTO IV-ACS randomised trial. Lancet. (2001). CrossRefMedline, 357, 1915-1924.

[10] Invasive compared with non-invasive treatment in unstable coronary-artery disease: FRISC II prospective randomized multicentre study: Fragmin and Fast Revascularization During Instability in Coronary Artery Disease (FRISC II) InvestigatorsLancet. (1999). CrossRefMedline, 354, 708-715.

[11] Outcome at 1 year after an invasive compared with a non-invasive strategy in unstable coronary-artery disease: the FRISC II invasive randomised trial: FRISC II Investigators: Fast Revascularisation During Instability in Coronary Artery DiseaseLancet. (2000). CrossRefMedline, 356, 9-16.

[12] Cannon, C. P, Weintraub, W. S, Demopoulos, L. A, et al. Comparison of early invasive and conservative strategies in patients with unstable coronary syndromes treated with the glycoprotein IIb/IIIa inhibitor tirofiban. N Engl J Med. (2001). CrossRefMedline, $344,1879-1887$.

[13] Berkowitz, S. D, Granger, C. B, Pieper, K. S, et al. for the Global Utilization of Streptokinase and Tissue Plasminogen Activator for Occluded Coronary Arteries (GUSTO) I Investigators. Incidence and predictors of bleeding after contemporary thrombolytic therapy for myocardial infarction. Circulation. (1997). Abstract/FREE Full Text, 95, 2508-2516.

[14] Fox KAAPoole-Wilson PA, Henderson RA, et al, for the Randomized Intervention Trial of Unstable Angina (RITA) Investigators. Interventional versus conservative treatment for patients with unstable angina or non-ST-elevation myocardial infarction: the British Heart Foundation RITA 3 randomised trial. Lancet. (2002). CrossRefMedline, 360, 743-751.

[15] Fox, K. A. A, Mehta, S. R, Peters, R, Zhao, F, Lakkis, N, Gersh, B. J, \& Yusuf, S. Benefits and Risks of the Combination of Clopidogrel and Aspirin in Patients Undergoing Surgical Revascularization for Non-ST-Elevation Acute Coronary Syndrome- The Clopidogrel in Unstable angina to prevent Recurrent ischemic Events (CURE) Trial; Circulation. (2004). , 110, 1202-1208.

[16] The Task Force on the use of antiplatelet agents in patients with atherosclerotic cardiovascular disease of the European Society of CardiologyEspert consensus document on the use of antiplatelet agents. Eur Heart J. (2004). PubMed], 25, 166-81.

[17] Guyatt, G, Schunëmann, H, Cook, D, et al. Grade of recommendation for antithrombotic agents. Chest. (2001). S-7S.[PubMed]

[18] Patrono, C, Coller, B, Fitzgerald, G. A, Hirsh, J, \& Roth, G. Platelet active drugs: the relationships among dose, effectiveness and side effects. Chest. (2004). S-64S.[PubMed]

[19] Servin, F. Low-dose aspirin and clopidogrel: how to act in patients scheduled for day surgery. Curr Opin Anaesthesiol. (2007). PubMed], 20, 531-4. 
[20] Kulik, A, Chan, V, \& Ruel, M. Antiplatelet therapy and coronary artery bypass graft surgery: perioperative safety and efficacy; Expert Opinion on Drug Safety[(2009). DOI: $10.1517 / 14740330902797081$

[21] Yusuf, S, Zhao, F, \& Mehta, S. R. Clopidogrel in Unstable Angina to Prevent Recurrent Events Trial investigators et al. Effects of clopidogrel in addition to aspirin in patients with acute coronary syndromes without ST-segment elevation, N Engl J Med CrossRef, 345-2001.

[22] Hongo, R. H, Ley, J, Dick, S. E, \& Yee, R. R. The effect of clopidogrel in combination with aspirin when given before coronary artery bypass grafting, J Am Coll Cardiol CrossRef, 40-2002.

[23] Yende, S, \& Wunderink, R. G. Effect of clopidogrel on bleeding after coronary artery bypass surgery, Crit Care Med CrossRef, 29-2001.

[24] Chen, L, Bracey, A. W, Radovancevic, R, et al. Clopidogrel and bleeding in patients undergoing elective coronary artery bypass grafting, J Thorac Cardiovasc Surg CrossRef, 128-2004.

[25] Englberger, L, Faeh, B, Berdat, P. A, Eberli, F, Meier, B, \& Carrel, T. Impact of clopidogrel in coronary artery bypass grafting, Eur J Cardiothorac Surg CrossRef, 26-2004.

[26] Fox, K. A. A, Mehta, S. R, Peters, R, et al. Benefits and risks of the combination of clopidogrel in patients undergoing surgical revascularization for non-ST elevation acute coronary syndromes. the Clopidogrel in Unstable Angina to Prevent Recurrent Ischemic Events (CURE) trial, Circulation CrossRef, 110-2004.

[27] Braunwald, E, Antman, E. M, Beasley, J. W, et al. ACC/AHA 2002 guideline update for the management of patients with unstable angina and non-ST-segment elevation myocardial infarction-summary article. a report of the American College of Cardiology/American Heart Association Task Force on Practice Guidelines (Committee on the Management of Patients With Unstable Angina), J Am Coll Cardiol CrossRef, 40-2002.

[28] Bhatt, D. L, Roe, M. T, Peterson, E. D, et al. Utilization of early invasive management strategies for high-risk patients with non-ST-segment elevation acute coronary syndromes. results from the CRUSADE Quality Improvement Initiative, JAMA CrossRef, 292-2004.

[29] Sonel, A. F, Good, C. B, Mulgund, J, et al. Racial variations in treatment and outcomes of black and white patients with high-risk non-ST-elevation acute coronary syndromes. insights from CRUSADE (Can Rapid Risk Stratification of Unstable Angina Patients Suppress Adverse Outcomes With Early Implementation of the ACC/AHA Guidelines?), Circulation , 111-2005.

[30] Albaladejo, P, Marret, E, Piriou, V, \& Samama, C. M. Perioperative management of antiplatelet agents in patients with coornary stents: recommendations of a French Task Force. Br J Anaesth. (2006). PubMed], 97, 580-84. 
[31] Dalal, A. R, Souza, D, \& Shulman, S. RS. Brief review: Coronary drug-eluting stents and anesthesia. Can J Anesth. (2006). PubMed], 53, 1230-43.

[32] Steinhubl, S. R, Bhatt, D. L, Brennan, D. M, Montalescot, G, Hankey, G. J, Eikelboom, J. W, Berger, P. B, \& Topol, E. J. CHARISMA Investigators. Aspirin to prevent cardiovascular disease: the association of aspirin dose and clopidogrel with thrombosis and bleeding; Annals of Internal Medicine[(2009).

[33] Mannacio, V. A. Di Tommaso L, Antignano A, De Amicis V, Vosa C; Aspirin plus clopidogrel for optimal platelet inhibition following off-pump coronary artery bypass surgery: results from the CRYSSA (prevention of Coronary arteRY bypaSS occlusion after off-pump procedures) randomised study Heart heartjnl-Published Online First: 2 September (2012). Heart doi:10.1136/heartjnl-2012-302449, 2012-302449.

[34] Elefteriades, J. A, \& Meier, P. Clopidogrel and cardiac surgery: enemy or friend?. Heart 2012; heartjnl-2012-302822Published Online First: 9 October (2012). doi:10.1136/ heartjnl-2012-302822, 98(20)

[35] Cannon, C. P, Mehta, S. R, \& Aranki, S. F. Balancing the benefit and risk of oral antiplatelet agents in coronary artery bypass surgery; The Annals of Thoracic Surgery[(2005).

[36] Rajendra, H, Mehta, M. D, Facc, M. S, Matthew, T, \& Roe, M. D. MHS, FACC; Jyotsna Mulgund, MS; E. Magnus Ohman, MD, FACC; Christopher P. Cannon, MD, FACC; W. Brian Gibler, MD; Charles V. Pollack, Jr, MD, MA; Sidney C. Smith, Jr, MD, FACC; T. Bruce Ferguson, MD; Eric D. Peterson, MD, MPH, FACC. Acute Clopidogrel Use and Outcomes in Patients With Non-ST-Segment Elevation Acute Coronary Syndromes Undergoing Coronary Artery Bypass Surgery J Am Coll Cardiol. (2006). doi:10.1016/ j.jacc.2006.04.029, 48(2), 281-286. 
